\title{
Is Case Volume the Only Surrogate for Oncologic Surgical Quality?
}

\author{
Frederick L. Greene, MD \\ Department of Surgery, Carolinas Medical Center, 1000 Blythe Blvd., MEB, Ste. 601, Charlotte, North Carolina 28203
}

The Editorial Board of the Annals of Surgical Oncology decided during the last year to develop a specific section of the Journal featuring studies that highlight the importance of well-done outcomes research pertinent to both the short- and long-term term surgical treatment of cancer. As editor of this section, it is particularly satisfying for me and the entire Editorial Board to include the work of Wouters and colleagues from the Netherlands Cancer Institute $^{1}$ in this month's publication. It is also rewarding for me to write a companion piece relating to this article since these authors confirm my particular biases, ${ }^{2}$ which include the concept that surgical case volume alone is not the only predictor of good outcome in the management of surgical treatment of cancer.

Wouters et al. have performed an in-depth study of the short- and long-term outcomes following resection for esophageal cancer both in low-volume and high-volume hospitals associated with the Comprehensive Cancer Center in Leiden. They have compared patients managed in 11 low-volume $(<7$ resections per year) hospitals to patients managed in a single high-volume center in Leiden. As previous studies have shown ${ }^{3,4}$ dealing with both esophageal and pancreatic cancer, the mortality and morbidity rates were significantly lower in the high-volume center. The authors, however, did not curtail their analysis at that point. They further initiated an

Received September 28, 2007; accepted October 1, 2007; published online: November 15, 2007.

Address correspondence and reprint requests to: Frederick L. Greene, MD; E-mail: frederick.greene@carolinashealthcare.org

Published by Springer Science+Business Media, LLC $\odot 2007$ The Society of Surgical Oncology, Inc. investigation of the individual patient comorbidities at the respective hospitals and showed that comorbidity was indeed a strong prognostic factor in predicting in-hospital mortality. This fact of course is not surprising, but the analysis of the case-mix data is frequently omitted from studies that look at volume as a singular predictor of quality. Furthermore, the authors analyzed the penultimate outcome in cancer management-5-year survival - and showed that at least for early-stage esophageal cancer (Stages I and II) there was a significantly better 5-year survival in the high-volume center.

This study once again confirms that the entire hospital environment is the most important predictor of patient outcome and that volume is but one indicator of quality in this equation. It is interesting that the authors made no mention of individual surgeon volume and performance, but related their outcomes to the total number of patients managed in the hospital environment. We are not told how many surgeons were involved in the 342 operations performed in the low-volume hospitals or in the 561 procedures performed in the high-volume centers. While this would be interesting, the important issues are overall care and the institutional infrastructure related to the management of cancer, not the case volume characteristics of the individual surgeons. It is assumed that even surgeons with a lower volume can have excellent results when they operate in an environment dedicated to good surgical support and cancer care.

It is obvious that in the discussion of any specific cancer, the outcome of the cancer patient relating to stage is an important characteristic. Wouters and colleagues looked not only at hospital volume, but 
also at patient, tumor, and treatment characteristics. Relating to esophageal management in the high-volume center, most patients were treated with transhiatal techniques with associated cervical anastomoses, while transthoracic procedures involving anastomoses in the chest were performed in low-volume centers. Obviously, choice of procedure is an important issue and morbidity resulting from varying techniques must be taken into account in any outcome study relating to volume. It was a little surprising, however, to see that positive margins of resection were actually higher in the high-volume centers. Since our mission as oncologic surgeons is to achieve R0 resections, certainly a benchmark of quality should be a high incidence of complete pathologic resection in any cancer operation. Perhaps the transhiatal approach contributed to the lower incidence of $\mathrm{R} 0$ resections in the high-volume centers. This fact, however, apparently did not affect the outcome especially in early-stage patients in centers with higher volumes.

There is no question that cancers of the pancreas, esophagus, and liver have been shown to have better outcomes when managed by surgeons with extensive experience in treating these lesions. ${ }^{3-5}$ Probably the most important indicator, however, as supported by the Dutch group, is that institutional volume plays a significant role in ensuring better patient outcomes in certain malignancies. The authors further tell us that dependence on large administrative databases for the purpose of assessing quality is fraught with danger. An example of this is a recent publication ${ }^{6}$ relating to increased mortality rates after lumpectomy in the treatment of breast cancer in "low-volume" hospitals. This study sends the wrong message and can adversely affect good surgeons who work in small- and medium-sized community hospitals.

Case volume has also surfaced as an important prognostic factor in the surgical management of benign abnormalities such as coronary artery disease and aortic aneurysms. It is hard to refute the notion that patients cared for by high-volume surgeons and high-volume institutions will inherently have better short-term and long-term outcomes. Although volume may be a structural measure of quality and "in-house" mortality may be an outcome measure, there continues to be a variable relationship between volume and outcome. There is no question that highvolume surgeons may indeed have poor outcomes. Quality standards relating to the entire institution are thus much more appropriate to have in place. The concept of risk adjustment must always be considered since this reduces the size of the volume effect between "low-" and "high-" volume hospitals and surgeons. This case mix may reflect the differences in socioeconomic levels of patients and the initial stage of cancers in populations treated by a given hospital and its surgical staff.

In my opinion, the paper by Wouters and colleagues should be used as a benchmark of solid outcomes research since it includes important indicators other than volume alone. Careful analysis of individual patients and their comorbidities is needed in these research models. The ultimate challenge for Wouters and his colleagues working in Holland is to export the excellence achieved in the Comprehensive Cancer Center and the specific high-volume hospital to all of the other eleven hospitals working in the affiliated system. I particularly look forward to the day when these researchers can tell me they have identified the benchmarks and have successfully transmitted these concepts to allow every patient with esophageal cancer to be managed equally in hospitals under their aegis!

\section{REFERENCES}

1. Wouters M, Wijnhoven B, Karim H, et al. High-versus lowvolume for esophageal resections for cancer: the essential role of case-mix adjustments based on clinical data. J Surg Oncol 2007; DOI 10.1245/s10434-007-9673-4.

2. Greene FL. Is volume the most important predictor of outcome in cancer management? J Surg Oncol 2007; DOI 10.1002/ jso.20833; Early View, published online July 26, 2007.

3. Killeen SD, O'Sullivan MJ, Coffey JC, et al. Provider volume and outcomes for oncological procedures. Br J Surg 2005; 92:389-402.

4. Finlayson EV, Goodney PP, Birkmeyer JD. Hospital volume and operative mortality in cancer surgery. Arch Surg 2003; 138:721-6.

5. Fong $\mathrm{Y}$, Gonen $\mathrm{M}$, Rubin D, et al. Long-term survival is superior after resection for cancer in high-volume centers. Ann Surg 2005; 242:540-7.

6. Guller U, Safford S, Pietrobon R, et al. High hospital volume is associated with better outcomes for breast cancer surgery: analysis of 233, 247 patients. World J Surg 2005; 29:9941000 . 